\title{
Factors Affecting Adherence to Treatment of HIV in Exposed Infants in Mumias Region, Western Kenya
}

\author{
Sophia Musenjeri ${ }^{1}$, Serah Mbatia ${ }^{1}$, Joseph Nganga $^{2}$, Matilu Mwau ${ }^{3}$ \\ ${ }^{1}$ Institute of Tropical Medicine and Infectious Diseases (ITROMID) Nairobi, Kenya, Jomo Kenyatta University of Agriculture and \\ Technology, Kenya Medical Research Institute, Nairobi, Kenya \\ ${ }^{2}$ Jomo Kenyatta university of Agriculture and Technology, Nairobi, Kenya \\ ${ }^{3}$ Consortium for National health research, Nairobi, Kenya
}

Email address:

namwasi.musenjeri@gmail.com (S. Musenjeri), serahmbatia@gmail.com (S. Mbatia),jnganga@jkuat.ac.ke (J. Nganga), matilu.mwau@gmail.com (M. Mwau)

\section{To cite this article:}

Sophia Musenjeri, Serah Mbatia, Joseph Nganga, Matilu Mwau. Factors Affecting Adherence to Treatment of HIV in Exposed Infants in Mumias Region, Western Kenya. Science Journal of Public Health. Vol. 3, No. 3, 2015, pp. 366-372. doi: 10.11648/j.sjph.20150303.20

\begin{abstract}
Objective: To determine social-demographic and economic factors affecting adherence to treatment of HIV in exposed infants in Mumias region, Western Kenya. Methods: The study was a descriptive cross sectional study carried out among parents of HIV exposed infants in selected health facilities in western Kenya. Through random sampling, the study recruited three hundred and eighty four (384) parents aged between 15-66 years old. The parents who were recruited were seeking HIV testing, treatment and care for their infants. The laboratory procedure involved automated assay: Abbott Real-time HIV-1. Secondly, structured interviewer administered questionnaire was used to collect information from parentsof the affected infants. Data was analyzed using SPSS version 20. Results: 5.2\% (20) of the participants tested positive while 94.8\% (364) tested negative. Married participants were more likely to adhere to treatment (Odds ratio (OR) $=1.062,95 \% \mathrm{CI} 0.628-1.796$ $\mathrm{P}<0.05)$. Educated participants were more likely to attend their clinical appointments compared to the non-educated $(0 \mathrm{R}=1.140,95 \%$ C.I $0.949-1.369 \mathrm{P}<0.05)$. Participants aged above 35 years old were more likely to adhere to treatment compared to those below 35 years old $(\mathrm{OR}=1.029,95 \%$ C.I $0.985-1.074 \mathrm{P}<0.05)$. Participants whose children tested negative at 6 weeks were more likely to adhere to treatment $(\mathrm{OR}=0.652,95 \%$ C.I $0.185-2.305 \mathrm{P}<0.05)$. Parentsunder the support of Community Health Workers $(\mathrm{CHW})$ were more likely to adhere to treatment $(\mathrm{OR}=1.226,95 \%$ C.I $0.419-3.581 \mathrm{P}<0.05)$. Nonstigmatized mothers were more likely to adhere to treatment $(\mathrm{OR}=1.101, \mathrm{P}<95 \%$ C.I $0.545-2.223)$. Conclusion: Adherence to treatment and care of HIV in exposed infants appears to be a significant challenge for HIV diagnostic and preventive services. To forestall the consequences, the stakeholders and government have to support the parents both financially and socially especially through public awareness campaigns to encourage them to adhere to treatment and care services.
\end{abstract}

Keywords: Adherence HIV, Infants, Diagnosis, Stigma, CHW

\section{Introduction}

In Sub-Saharan Africa,Human Immunodeficiency Virus (HIV) infection is prevalent in women who are in their reproductive age ${ }^{[1,2]}$. In 2009, the Kenya National AIDS and STD Control Programme (NASCOP) estimated that $5.6 \%$ of pregnant women were HIV positive while in 2010 more than 80,000 pregnant women were HIV positive. Mother to Child Transmission (MCT) of HIV accounted for 10,390 infants' deaths in $2013^{[3,4]}$. The Ministry of Health $(\mathrm{MoH})$ in Kenya established an Early Infant Diagnosis (EID) program to reduce HIV infection in infants. All exposed infants start taking septrine immediately after birth. The HIV DNA Polymerase Chain Reaction (PCR) determines HIV infection in infants aged between 6 weeks and 24 months. The EID program uses the dried blood spots technology and automatedAbbott real time HIV-1 or automated Cobas ${ }^{\circledR}$ AmpliPrep / Cobas ${ }^{\circledR}$ TaqMan ${ }^{\circledR}$ HIV-1 to determine the HIV status of exposed infants ${ }^{[5,6]}$. Therapeutic and prophylactic medications are initiated timely for infants ${ }^{[7]}$.

Infants who are HIV positive start on cotrimoxazole prophylaxis at six weeks ${ }^{[8]}$. Due to continuous exposure, infants who test negative but their mothers are positive are also initiated on cotrimoxazole prophylaxis ${ }^{[4,9]}$. Follow up is done on the exposed infants under medication. At the age of 
twenty-four months, the infants are re-tested to ascertain their HIV status.

Prevention of MCT of HIV relies heavily on consistence in antiretroviral therapy ${ }^{[10]}$. The speculation is that at least $50 \%$ of the mothers of the exposed infants get their results almost timely; however, some of them do not. Although those who receive positive results can get into free treatment and care, not all do. Unfortunately, the reasons, which motivate an individual to either get into treatment or not are yet to be known. The Ministry of Health in Kenya has established many EID sites to increase access. Therefore, many exposed infants are tested for HIV and treatment initiated immediately. However, new child infections of HIV and negligence in treatment have serious implications on reducing the disease burden and child mortality.

The aim of the study was to establish the factors affecting entry into care and treatment for HIV in exposed infants in Mumias District, Western Kenya.

\section{Methods and Materials}

\subsection{Study Design and Setting}

An institution based quantitative cross-sectional study was carried out in major EID sites in Mumias region, Western rural Kenya. Mumias is situated about $416 \mathrm{~km}$ from Nairobi. Ideally, the ministry of health has picked a few health facilities to serve as Early Infant Diagnosis (EID) sites, which include St.Marys Mission hospital, Matungu subdistrict hospital, Mumias sugar clinic, and Mumias model dispensary, Bukaya dispensary, Makunga dispensary and Malaa health centre. The health facilities in the region combine both child wellness and Early Infant Diagnosis program.

\subsection{Study Participants}

The participants were both male and female aged 15-66 years old. All the participants were HIV positive parents whose infants had been exposed to HIV in utero. The infants were attending HIV testing, treatment and care.

\subsection{Sample Size and Sampling Techniques}

To calculate the sample size, Cochran 1963 formula $[n=$ $\left.Z^{2} P(1-P) / e^{2}\right]$ was used. Proportion of 0.5 was applied and a confidence interval of $95 \%$

Whereby $n$ was the sample size,

$Z^{2}$ was the abscissa of the normal curve that cuts off an area at the tails (1- equals the desired confidence level, which was $95 \%$ ),

$e$ was the desired level of precision (80\%)

$p$ was the estimated proportion of an attribute that is present in the population.

The value for $Z$ was found in statistical tables, which contain the area under the normal curve (1.96); therefore, a sample of 384 was used during the study. The first 384 parent-infant pair who agreed to participate in the study were interviewed one after the other regardless of the health facility.

\subsection{Data Collection}

Data was collected using self-administered close-ended questionnaire. The questionnaire was prepared in English and the local dialect (luhya) commonly used in the region. The contents of the questionnaire included social, economic and demographic characteristics that may affect adherence to treatment and care of infants under EID. Before the commencement of the study, the questionnaire was pretested on at least $8 \%$ of the population attending EID treatment and care.The importance of the pretesting was to check on the ability of participants to comprehend the questions, the duration the interview will take, language comprehension and structuring of the questions. After pretesting, the questionnaire underwent slight modification to fit the study population. In addition, Cronbach's alpha test was used to validate and confirm the data collection tool. The data was collected everyday for a period of six months.

\subsection{Clinical Specimens}

Using filter papers, dotted blood spots (DBS) were collected from the participant's infants by the nurse incharge.

\subsection{Laboratory Analysis}

For automated PCR assay, Abbott Real time HIV-1 tested the HIV status of the infants. Using a clean, disinfected pair of forceps, two $2 \mathrm{~mm}$ discs were excised from the pre-cut Dried Blood Sample and carefully placed into labeled sterilized tubes ${ }^{[11]}$. The procedure was done inside a biosafety class II cabinet. Disinfection of the pair of forceps was done using 10\% sodium hypochlorite, distilled water and $70 \%$ ethanol respectively. Disinfection was done after cutting each sample to prevent cross-contamination between samples. The above procedure was repeated until all samples had been transferred into their respective falcon tubes. Approximately, 1760 microlitres of the bulk lysis (specimen extraction buffer) was added in each falcon tube-containing sample. The prepared sample was incubated for 30 minutes. After incubation, reaction tubes were labeled in correlation with the falcon tubes. Approximately 1760 microlitres of the extracted specimen was transferred into the respective reaction tubes placed in Abbott racks. The Abbott instrument has six racks, each with ability to hold 16 samples. Thus, the total samples tested at a time were 96 . However, the last two slots of the last rack were placements for positive and negative controls. The racks were then loaded onto the Abbott sample preparation instrument fully loaded with the reagents, consumables for proviral DNA extraction. Amplification and detection was through manual transfer of extracted DNA to Abbott real time amplification instrument. Results were indicated as either target detected for positive or target not detected for the negative results. All positive samples were re-tested for confirmatory purposes. 


\subsection{Data Analysis}

Data was entered and analyzed using statistical package for windows SPSS version 20. 0. Descriptive statistics was used to summarize and present data in form of percentages or frequencies. Odds ratio $(95 \% \mathrm{CI})$ was used to analyze the association between social-demographic variables and level of adherence of the participants.

\subsection{Ethical Considerations}

Participants signed consent forms as per the guidelines of the Kenya National Ethical Review Committee. Scientific approval was sought from the Scientific Steering Committee at Kenya Medical Research Institute (KEMRI).

\section{Results}

The study was carried out in a period of 6 months, from June 2014 to December 2014. 5.2\% (20) of the infants tested were positive while $94.8 \%$ (364) tested negative. 75 (20.6\%) of the Infants who tested negative missed at least one or more appointments while $30 \%$ (6) of those who tested positive missed one or more of their monthly appointments.

\subsection{Social and Demographic Features of the Participants}

Using random sampling, 384 participants whose infants had been tested for HIV during the study period were recruited into the study. All the participants responded to the questionnaire. The sample was composed of $97.9 \%$ females and $2.1 \%$ men. The participants had a mean age of 32.82 and standard deviation of 6.870 and standard error of 0.351 . Furthermore, the median age was 33 while mode age 32 , the age ranged from 15-66. In terms of marital status, at least $92.7 \%$ of the participants were married while the rest were single, divorced or widowed (Table 1). More than half of the participants had at least attended primary school with the rest either lacking formal education or having not completed primary education. More details in Table 1 . Nearly half of the $43.75 \%$ participants were either in self/informal employment or in formal employment while the rest were casual workers or not employed (Table 1). The participants were either Christians 66.66\% (256) or Muslims 33.33\% (122) (Table 1).

Table 1. Social-economic and demographic characteristics.

\begin{tabular}{|c|c|c|c|}
\hline Characteristic & & $\mathrm{N}=384$ & Percentage \\
\hline \multirow{4}{*}{ Marital status } & Never married & 28 & $7.3 \%$ \\
\hline & Married & 328 & $85.4 \%$ \\
\hline & Separated & 12 & $3.1 \%$ \\
\hline & Widowed & 16 & $4.2 \%$ \\
\hline \multirow{5}{*}{ Level of education } & No formal education & 48 & $12.5 \%$ \\
\hline & Primary incomplete & 118 & $30.7 \%$ \\
\hline & Primary complete & 89 & $23.2 \%$ \\
\hline & Secondary complete & 48 & $12.5 \%$ \\
\hline & Tertiary complete & 31 & $8.1 \%$ \\
\hline \multirow{5}{*}{ Occupation } & No answer & 1 & $0.3 \%$ \\
\hline & Salaried formal employment & 31 & $8.1 \%$ \\
\hline & Informal employment & 40 & $10.4 \%$ \\
\hline & Self employment & 97 & $25.3 \%$ \\
\hline & Casual worker & 48 & $12.5 \%$ \\
\hline \multirow{2}{*}{ How does the society treat you and your child } & Stigmatized & 276 & $71.9 \%$ \\
\hline & Supportive & 108 & $28.1 \%$ \\
\hline \multirow{3}{*}{$\begin{array}{l}\text { Financial challenges you incur as a parent under } \\
\text { this program. }\end{array}$} & & 3 & $0.8 \%$ \\
\hline & Yes & 322 & $83.9 \%$ \\
\hline & No & 59 & $15.4 \%$ \\
\hline \multirow{2}{*}{ Do you have other children } & Yes & 368 & $95.8 \%$ \\
\hline & No & 16 & $4.2 \%$ \\
\hline \multirow{7}{*}{ How much do you earn in KShs? } & No income & 191 & $49.7 \%$ \\
\hline & Below 500 & 17 & $4.4 \%$ \\
\hline & $501-1000$ & 35 & $9.1 \%$ \\
\hline & $1001-2000$ & 35 & $9.1 \%$ \\
\hline & $2001-3000$ & 26 & $6.8 \%$ \\
\hline & Above 3001 & 80 & $20.8 \%$ \\
\hline & & 2 & $0.5 \%$ \\
\hline
\end{tabular}

\subsection{Factors Affecting Adherence to Treatment of HIV in Exposed Infants}

Various factors either positively or negatively affect the uptake treatment and care.

The major challenges that face the primary care givers include financial constraints whereby $83.9 \%$ (322) attributed it as the reason to why they sometimes have to miss their clinic appointments. $76.3 \%$ (293) of the interviewees had to have at least Ksh.50 and above in order to access treatment in the nearest health centre.

Secondly, societal stigma is a problem that affects 276 $(71.88 \%)$ of the participants while 108 (28.12\%) of the interviewees attribute their success to the community and 
family members support. Similarly, support from community health workers encouraged participants to seek treatment and care. Only Matungu Sub-district hospital employed the services of community health workers (CHW). Ironically, only 22 participants in Matungu health centre acknowledged being visited by $\mathrm{CHW}$.

Due to inconsistent supply of ARV's 25\% (29) Participantsof the interviewees at St.Mary's Mission Hospital missed their doses during the study period. The nurse inchargeadvised them to divide adult NVP into half and give to the infants.

Nearly half of the participant $191(49.7 \%)$ have $0 \%$ monthly income while $113(79.13 \%)$ of the participants earn less than 3000 Kenyan shillings in a month. The rest of the participants $80(20.87 \%)$ earn more than 3000 shillings in a month.

At least 94\% (361) of the participant had received education on the important of Early Infant Diagnosis. However, 5.2\% (20) admitted to not having any knowledge about the program, more details given in Table 2 .

Table 2. Factors affecting adherence to treatment and care.

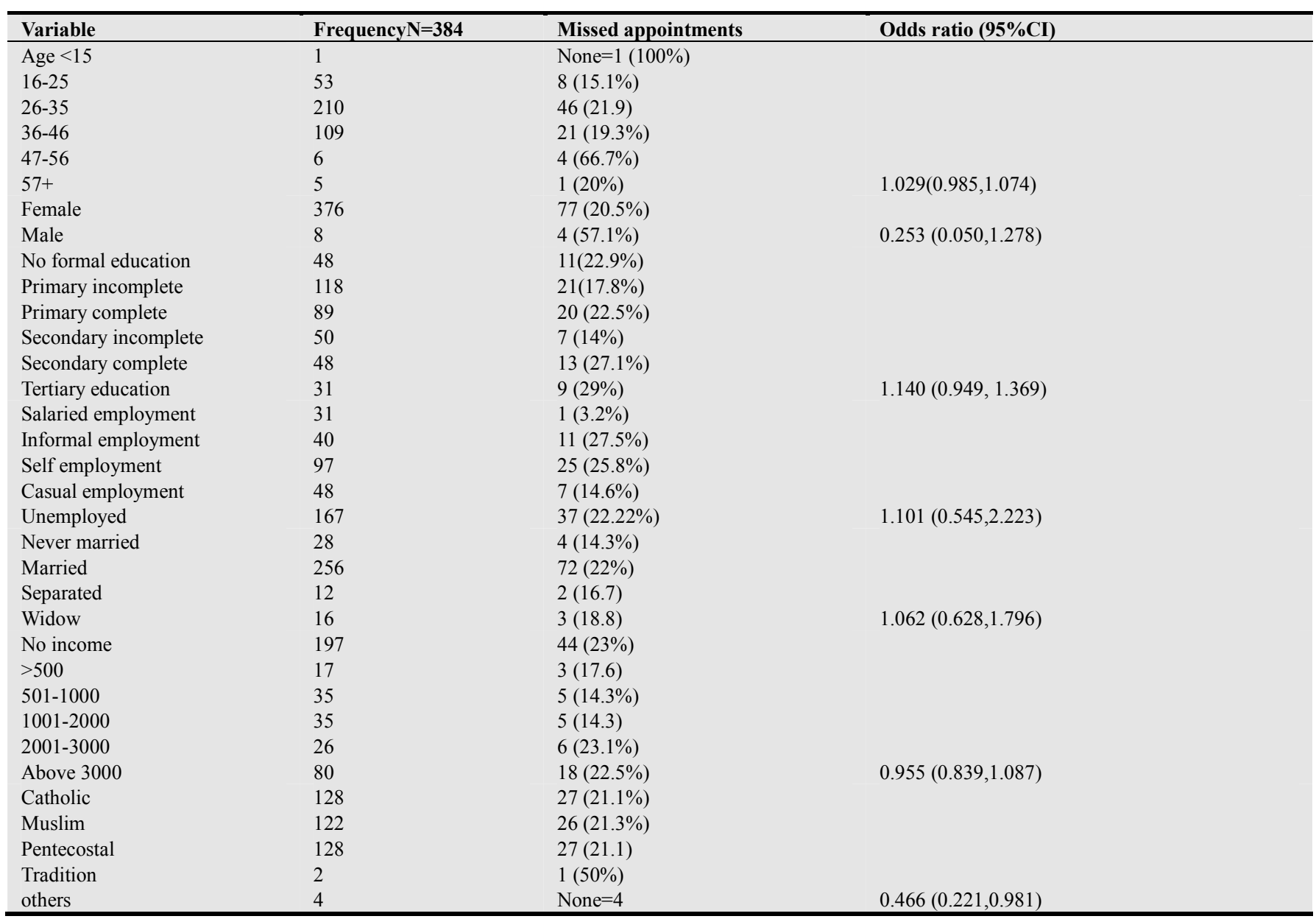

$P$ value $=0.5$

A chi-square test was used to test the independence between socio-demographic factors and the number of missed appointments. The significance level was reported when $\mathrm{p}<0.05$. Table 3 shows the socio-demographic factors that were significant relation to adherence level of the participants.

\subsection{Level of Adherence Among Participants}

Adherence level was measured in terms of number of missed appointments recorded in a period of six months during the study. At least $21.1 \%$ (81) of the participants missed either one or more appointments. The participant with the poorest level of adherence missed all thefour appointments $(1.23 \%)$

\section{Discussion}

In Kenya, the prevalence of HIV in women is estimated by NASCOP to be $7.6 \%$, which translates to new HIV infections in newborns. Although EID has reduced PMTCT to approximately $3.5 \%$ in developed countries [12], there is limited data showing its impact among infants and children in developing countries including Kenya. The Kenya AIDSIndicator Survey estimated in 2013 that 12,940 children were newly infected ${ }^{[4]}$. In relation to these reasons, interventions to encourage parents to enroll in EID will reduce new HIV infections in children. Timely management 
of HIV exposed infants minimizes the risk of vertical transmission. Maternal adherence to treatment during pregnancy plus consistent use of neonate zidovudine greatly reduces infection of HIV to newborns ${ }^{[13]}$. On the other hand, social, demographic and economic factors work synergistically to make adherence to treatment and care of
HIV in infants difficult. This study established a strong correlation between socio-demographic factors and uptake of treatment. From the results above, there is a significant relationship between stigmatization, EID education, occupation, presence of other infected children and the frequency of missed appointments (see table 3).

Table 3. Association between social-demographic factors and adherence to treatment.

\begin{tabular}{|c|c|c|c|c|}
\hline \multicolumn{5}{|l|}{ Chi-Square Tests } \\
\hline Characteristic & Value & df & Frequency & Asymp. Sig. (2-sided) \\
\hline Stigmatization & 8.082 & 1 & $\mathrm{~N}=276(71.9 \%)$ & 0.004 \\
\hline Presence of other children & 8.819 & 2 & $\mathrm{~N}=368(95.8 \%)$ & 0.012 \\
\hline Other children (HIV+) & 8.381 & 1 & $\mathrm{~N}=43(11.2 \%)$ & 0.004 \\
\hline Education on EID & 6.379 & 2 & $\mathrm{~N}=361(94 \%)$ & 0.041 \\
\hline Occupation & 9.525 & 4 & $\mathrm{~N}=216(56.3 \%)$ & 0.049 \\
\hline Total sample size $=384$ & & & & \\
\hline
\end{tabular}

From the study,most of the participants were female $97.1 \%$. This is evident that the responsibility and burden of taking care of a sick child is always left to the mother. Various studies have established that men rarely seek health care unless they are in critical condition ${ }^{[14]}$. Due to AIDS related deaths, some of the infants had been orphaned and thus the responsibility of taking care of them had been left to their grandmothers.

The age of participants directly affected their level of adherence to treatment. For instance, an increase in age increased the chances of a mother-infant pair getting into treatment and care by at least $2.9 \%(\mathrm{OR}=1.029,95 \%$ C.I 0.985-1.074 $\mathrm{P}<0.05)$. However, this was not the case for the old, poor and weak grandmothers. Mothers below 35 years of age were more likely to miss clinical appointments when compared to their older counterparts. Married mothers were $6 \%$ more likely to adhere to clinical appointments compared to non-married ones.

A study conducted inKakamega district by the University of Nairobi, established that educationstatus, economic ability of the HIV positive mothers and their occupation affects entry into care and treatment ${ }^{[15]}$. Education status of the caregiver boosts the adherence level of positive mothers. However, from the study only $56.7 \%$ of the caregivers had completed their primary education. Consequently, parents who had at least completed their primary education were $13.1 \%$ more likely to adhere to clinical appointments and treatment compared to those who had little or no formal education at all. The ministry of health in Kenya ensures that every person who seeks HIV treatment undergoes training on how to treat and manage the disease. This study established that (361) $94 \%$ of the participants had knowledge about EID. Therefore, participants who had knowledge on EID were 3.9\% less likely to miss their clinical appointments $(\mathrm{OR}=0.962,95 \%$ $\mathrm{P}<0.05$ 0.296-3.130).Lack of knowledge on EID promotes ignorance and poor attitude towards accessing and adherence to treatment and care of HIV[16].

Participants who earn between KSh. 0 to 1000 per month are $4.6 \%$ more likely to miss an appointment compared to those who earn KSh. 1000 and above monthly (OR=0.955
95\% C.I 0.839-1.087 $\mathrm{P}<0.05)$. Similarly, according to Muchedzi et al., the economic status of the family determined their priority to access basic needs like food, shelter and health is not among them ${ }^{[17]}$. In 2010, UNICEF stated high poverty levels as a factor that negatively contributes to accessibility to health centers ${ }^{[18]}$.

At least $71.88 \%$ of the participants in the study face stigmatization; this hindered them from enrolling into treatment and care. Adherence to treatment and clinical becomes poor yet the children have to take their drugs to minimize chances of drug resistance ${ }^{[19]}$. Parents, who receive some form of support from either the extended family or community, are $19.1 \%$ more likely to comply with their clinic appointments without failure compared to their counterparts who received no form of support from their surrounding $(\mathrm{OR}=1.211,95 \% \quad \mathrm{P}<0.05$ C.I $0.886-1.654)$. Other issues stated include cultural and behavioral practices, which forces them to keep their HIV status a secret.

Any form of support from the health centre other than treatment produced positive effects in compliance with enrollment into care and treatment. For instance, a community health worker visit to the home of a respondent was significant; this increased their chance of attending to all the scheduled clinic appointment by $20.3 \%$ unlike their counterpart respondents who never had any community health worker's visit to their homes. Thus, CHW motivate parents to enroll infants in EID.

Muchedzi et al, mentions the quality of services offered to mother-infant pair as either a motivating or demoralizing factor ${ }^{[17]}$. According to health workers, poor turnaround time (more than 2 months), and communication breakdown with the reference laboratories has contributed to low turn up of patients after EID. Shortage of medical supplies forces the health workers to turn away the patients, which eventually demoralizes them from enrolling into treatment and care. This has also contributed to high number of defaulters.

Health workers ascertained that more than $50 \%$ of mothers come back for results. This is due to their anxiety to know the HIV status of their children. Similarly, according to NASCOP, in 2010, only $64 \%$ of HIV exposed infants received PCR test. Furthermore, only $7 \%$ of them proceed to 
treatment ${ }^{[2,20]}$. In the study at least, 10 defaulters were interviewed during the study and they pointed at various social and economic factors that hinder them from seeking their results from the health centers. For instance, they raised delay of results and lack of communication as to when the results will be delivered from KEMRI as a demoralizing factor. Lack of support from spouse and otherfamily members leaves all the burden ofcaring for a HIV positive child to the mother yet she is unable to cater for all the family needs. A visit by a health worker increased enrollment and consistency in adhering to treatment and care by $20.3 \%$ (Table 2). In 2010 UNICEF, Kenya stated lack of education awareness on HIV, communication barriers, poverty, behavioral and cultural as factors that negatively affect enrollment of infants into EID ${ }^{[18]}$. In addition, other issues stated include cultural and behavioral practices, which forces care givers especially a mother to keep their HIV status a secret.

\section{Conclusion}

In 2013, HIV accounted for 10390 infants' deaths in Kenya. Although the ministry of health in Kenya provides free treatment to HIV exposed infants, the mortality rates are yet to drop. The study established a significant correlation between social, demographic and economic factors versus adherence to treatment and care. Some of these factors include education level, marital status, occupation, income level, gender, presence of other children, support from the communities and stigmatization among others. Support from the CHW and family motivated the participants to seek and be consistency in EID. The quality of services offered to EID participants ensured either consistence or inconsistency in treatment. Furthermore, a breakdown of social relationships in the society has left the responsibility of taking care of HIV affected infants to mothers. Due to the burden, mothers shun health services, which include EID. In terms of education only $12.4 \%$ of the participants at least attended secondary school. Due to ignorance, most parents take EID education less seriously, which contributes to poor enrollment into treatment and care after EID. Whilst antiretroviral therapy drugs are readily available, the uptake of drugs and adherence to treatment is yet to reach $100 \%$. There is need by policy makers to understand the impact of the social description of an individual to EID

The greatest limitation of the study resulted from the fact that most of the defaulters could not be traced. This study highlights the major setbacks the EID stakeholders should focus on.

\section{Acknowledgment}

We appreciate everybody who participated in the study especially the participants. The study was conducted in conjunction with Kenya Medical Research Institute, Centre for Infectious and Parasitic Diseases.

\section{References}

[1] NASCOP. National Guidelines for Antiretroviral Therapy in Kenya, 4th Edition. In. Nairobi, Kenya; 2011.

[2] NASCOP Na. Kenya AIDS Epidemic update; 2012. In; 2012.

[3] NASCOP. Kenya AIDS Indicator Survey 11-Final report. http://nascop.or.ke/library/3d/Preliminary\%20Report\%20for $\% 20$ Kenya\%20AIDS\%20indicator\%20survey\%202012.pdf 2013:7-14.

[4] NASCOP. Kenya AIDS progress report 2014. http://www.unaids.org/sites/default/files/country/documents/K EN_narrative_report_2014.pdf 2014.

[5] Khamadi S. OV, Lihana R., Nabwera J., Hungu J., and Okoth F., . Rapid identification of infants for antiretroviral therapy in a resource poor setting: the Kenya experience. Journal of Tropical Pediatrics 2008,54:370-374.

[6] Zijenah LS. HJ, Nathoo K Evaluation of the prototype Roche DNA amplification kit incor- 5 porating the new SSK145 and SKCC1B primers in detection of human immunodeficiency virus type 1 DNA in Zimbabwe. Journal of Clinical Microbiology 1999,37:3569-3570.

[7] Cassol S.A. LN aST. Diagnosis of vertical HIV-1 transmission using the polymerase chain reaction and dried blood spot specimens. Journal of Acquired Immune Deficiency Syndrome 1992,113 .

[8] Liu L JH, and Cousens S. Global, regional, and national causes of child mortality: an updated systematic analysis for 2010 with time trends since 2000. lancet 2012,27:22-26.

[9] NASCOP. Kenya HIV Drug resistance Country Report. In. Nairobi; 2011.

[10] Creek T. TA SM. Early diagnosis of human immunodeficiency virus in infants using 105 polymerase chain reaction on dried blood spots in Botswana's national program for prevention of mother-to-child transmission. Pediatrics Infecioust Diseases journal 2008,27:22-26.

[11] Albert J aBIAs, sensitive, and specific detection of human immunodeficiency virus type 1 subtype B DNA in dried blood samples for diagnosis in infants in the field. . simple,sensitive, and specific detection of human immunodeficiency virus type 1 subtype B DNA in dried blood samples for diagnosis in infants in the field. Journal of Clinical Microbiogy. 2 2001,39:29-33.

[12] Audu R A. SOB, Musa AZ Estimation of the rate of mother to child transmission of HIV in Nigeria. African Journal of Medical science 2006,35:121 to 124 .

[13] ALEX H. KRIST MD, and AMY CRAWFORD-FAUCHER. Management of Newborns Exposed to Maternal HIV Infection. American Academy of Family Physicians 2002,65::049-056,2061.

[14] Galdas PM CF, and Marshall P. . Men and health help-seeking behaviour. journal of Adv NVPS 2005,49.

[15] Omondi MP. An assessment of institutional factors affecting uptake of ARV prophylaxis amongst HIV positive pregnant women in Kakamega District. In: University of Nairobi; 2010. pp. 14. 
372 Sophia Musenjeri et al.: Factors Affecting Adherence to Treatment of HIV in Exposed Infants in Mumias Region, Western Kenya

[16] N T. Sexual activity of out-of-school youth, and their knowledge and attitudes about STDs and HIV/AIDS in southern Ethiopia. Ethiopian Journal of Health Development. 1998,12:17-22.

[17] Muchedzi A. CW KJ, Stranix-Chibanda L., Woelk G., Mbizvo E., Shetty A K. Factors associated with access to HIV care and treatment in a prevention of mother to child transmission programme in urban Zimbabwe. Journal of the International AIDS Society 2010,13.

[18] World Health Organization JUNPoHA UNCsF. Towards Universal Access: Scaling up Priority HIV/AIDS Interventions in the Health Sector. In. http://www.unaids.org/sites/default/files/country/documents/K EN_narrative_report_2014.pdf.; 2011.
[19] Newell ML. CH CBM, Rollins N., Gaillard P., Dabis F. Ghent International AIDS Society (IAS) Working Group on HIV Infection in Women and Children. Mortality of infected and uninfected infants born to HIV-infected mothers in Africa: a pooled analysis. lancet kenya 2004,364:1236-1243.

[20] Programme NAaSC. Kenya AIDS Indicator Survey 2012: Preliminary Report, Nairobi, Kenya. In: Ministry of Health; 2013. 